\title{
Associação da dieta de cafeteria com amido resistente na modulação do perfil glicêmico em ratos Wistar
}

\section{Association between the cafeteria diet with resistant starch in the modulation of the glycemic profile in Wistar rats}

\author{
Tamires Aline Oliveira ${ }^{1}$, Rafael de Paula Alves ${ }^{1}$, Thaiany Goulart \\ de Souza e Silva ${ }^{2}$, Hudsara Aparecida de Almeida Paula ${ }^{3}$
}

Resumo

\begin{abstract}
A dieta hipercalórica é considerada fator de risco para o desenvolvimento de doenças cardiovasculares, sendo notado que os hábitos ocidentais possuem uma grande influência no desenvolvimento dessas enfermidades. A biomassa e a farinha de banana verde são alimentos que contém amido resistente; este constitui um tipo de carboidrato não digerível categorizado como prebiótico, com papel de regulação da glicemia. O principal objetivo deste estudo foi avaliar o efeito modulador dos alimentos supracitados em ratos Wistar alimentados com dieta de cafeteria. Foi possível observar uma grande diferença na composição centesimal e de amido resistente entre os dois prebióticos, sendo explicada pelo método de fabricação de cada um. Os grupos de intervenção contendo amido resistente proveniente da biomassa/farinha de banana verde demonstraram, no geral, um melhor perfil glicêmico, menor peso corporal e menor gordura corporal. O consumo alimentar com composição similar à dieta de cafeteria corriqueiramente pode levar a danos cardiometabólicos a longo prazo, contribuindo para o desenvolvimento de doenças crônicas. Observou-se também que as matrizes alimentares que contém amido resistente auxiliam no controle do ganho de peso corporal, prevenindo o acúmulo de tecido adiposo e intervindo no manejo dos níveis glicêmicos. Em suma, destaca-se que a implementação dos produtos da banana verde na alimentação pode ser de grande valia, considerando os efeitos positivos de seus componentes bioativos, ação prebiótica e a relação custo benefício desta matriz alimentar; porém, mais estudos científicos são necessários para confirmar as demais hipóteses que surgem com o tema.
\end{abstract}

Palavras-chave: Prebióticos. Glicemia. Hipercalórico.

1 Graduandos em Nutrição pela Universidade Federal de Alfenas, UNIFAL - MG, Alfenas, Minas Gerais, Brasil.

2 Doutoranda do Programa de Pós-Graduação em Biologia Celular pela Universidade Federal de Minas Gerais, Belo Horizonte, Minas Gerais, Brasil.

3 Doutorado em Ciência e Tecnologia de Alimentos pela Universidade Federal de Alfenas, UNIFAL - MG, Alfenas, Minas Gerais, Brasil. Professora Adjunta do Curso de Nutrição da Universidade Federal de Alfenas, UNIFAL - MG, Alfenas, Minas Gerais, Brasil. E-mail: hudsarald@gmail.com 


\section{Abstract}

The hypercaloric diet is considered a risk factor for the development of cardiovascular diseases, and it is noted that western habits have a great influence on the development of these diseases. Biomass and green banana flour are foods containing resistant starch; this is a type of non-digestible carbohydrate categorized as prebiotic, with the role of glycemia regulation. The main objective of this study was to evaluate the modulating effect of the aforementioned foods on Wistar rats fed with a cafeteria diet. It was possible to observe a great difference in the centesimal composition and resistant starch content between the two prebiotics, being explained by the manufacturing method of each one. Intervention groups containing resistant starch from biomass/green banana flour showed a better glycemic profile, lower body weight and lower body fat. Food consumption with composition similar to the cafeteria diet can lead to long-term cardiometabolic damage, contributing to the development of chronic diseases. It was also observed that food matrices containing resistant starch help to control body weight gain, preventing the accumulation of adipose tissue and intervening in the management of glycemic levels. In summary, the implementation of green banana products in food can be of great value, considering the positive effects of its bioactive components, prebiotic action and the cost-benefit ratio of this food matrix, but more scientific studies are needed to confirm the other hypotheses that arise with the theme.

Keywords: Prebiotics. Glycemia. Hypercaloric.

\section{Introdução}

A dieta hipercalórica é considerada fator de risco para o desenvolvimento de doenças cardiovasculares, sendo preocupante para a saúde pública mundial como exibido por Marques. ${ }^{(1)}$ Sabe-se que fatores relacionados ao estilo de vida ocidental, como certos padrões alimentares, sedentarismo e estresse psicossocial, associados à predisposição genética, contribuem para deposição de gordura visceral, geradora de resistência à insulina e eventos cardiometabólicos nocivos. O papel da dieta na gênese dessas doenças é tema de interesse global. ${ }^{(2)}$

A dieta de cafeteria, também conhecida como dieta ocidentalizada, baseia-se em alimentos industrializados, de grande densidade energética, ricos em carboidratos simples, lipídeos, sal e proteína animal e, simultaneamente, baixa ingestão de vegetais. Este modelo mimetiza uma refeição fast-food e tem sido bem aceito como um dos modelos que mais se aproximam da obesidade humana. ${ }^{(3)}$

Dentre as condições limitantes para risco cardiometabólico, encontra-se o sobrepeso/ obesidade, resistência à insulina, dislipidemia e hiperglicemia. ${ }^{(4)}$ Entre estes fatores, observam-se altas chances de um perfil glicêmico desregulado em indivíduos com sobrepeso/obesidade, assim como o maior risco do desenvolvimento de diabetes mellittus tipo 2 quando o perfil glicêmico está constantemente desequilibrado.

Desta forma, a obesidade está diretamente associada às dislipidemias e ao diabetes mellitus tipo 2. Sendo assim, tais doenças impõem diversas metas terapêuticas aos profissionais de saúde e ao indivíduo enfermo. Entre elas, a mudança do hábito alimentar, a inclusão da prática de atividades físicas e também o tratamento farmacológico. ${ }^{(2,5)}$

Em relação ao hábito alimentar, a busca pelo maior consumo de alimento funcional tem despertado atenção não somente da comunidade científica, mas também da população, que visa manter o estado nutricional saudável e reduzir o risco ou retardar o aparecimento de doenças. Tendo em vista o crescente interesse das pessoas por este tipo de alimento, criou-se um cenário de aumento da procura e consequente desenvolvimento de novos produtos/ingredientes pelas indústrias do segmento alimentício. ${ }^{(6)}$ 
O amido resistente, presente na biomassa e na farinha de banana verde, constitui um tipo de carboidrato não digerível categorizado como prebiótico que apresenta funcionalidade frente a diversos distúrbios orgânicos, aqui com destaque para a regulação do perfil glicêmico. Este ingrediente quando associado à alimentação pode contribuir, de forma sinérgica, para minimizar os efeitos nocivos da dieta ocidentalizada.

A partir da problemática apresentada e os possíveis benefícios que a biomassa e a farinha de banana verde podem oferecer, é relevante destacar a importância da proposta apresentada, em modelo animal, para maiores elucidações que possam auxiliar no manejo do perfil glicêmico.

\section{Material e Métodos}

\section{Aquisição da biomassa e farinha de banana verde}

A aquisição dos ingredientes biomassa e farinha de banana verde foi realizada no comércio local do município de Alfenas - MG.

\section{Determinação da composição centesimal e teor de amido resistente}

A determinação da composição centesimal e do teor de amido resistente foram realizadas pelo Centro de Raízes e Amidos Tropicais (CERAT), da Universidade Estadual Paulista Campi Botucatu, seguindo a metodologia $\mathrm{AOAC}^{(7)}$ e Goni et al. ${ }^{(8)}$

\section{Animais experimentais}

Foram utilizados 24 ratos (Rattus norvegicus, variedade albinus, classe Rodentia), da raça Wistar, recém-desmamados (21 dias de idade) provenientes do Biotério Central da Universidade Federal de Alfenas.

Os animais foram mantidos em gaiolas individuais de aço inoxidável, em ambiente de temperatura $\left(22 \pm 2^{\circ} \mathrm{C}\right)$ e luz controladas, em fotoperíodo de 12 horas e receberam água potável ad libitum.

\section{Delineamento experimental}

O experimento foi conduzido durante $o$ período de 20 dias. Os animais foram alocados de maneira randômica em 4 grupos $(n=6)$ : Controle (C), Dieta de cafeteria (CAF), Dieta de cafeteria associada a biomassa de banana verde - $8 \%$ de amido resistente $(\mathrm{CAF}+\mathrm{B})$ e Dieta de cafeteria associada a farinha de banana verde - $8 \%$ de amido resistente $(\mathrm{CAF}+\mathrm{F})$. $\mathrm{O}$ teor de amido resistente utilizado fundamentou-se no trabalho de Weaver et al. ${ }^{(9)}$ para evitar efeitos gastrointestinais indesejáveis.

\section{Dietas experimentais}

Para o desenvolvimento das dietas experimentais primeiramente foi realizado o preparo das dietas de cafeteria padronizadas, ${ }^{(10)}$ servindo estas então como base para a associação dos prebióticos biomassa e farinha de banana verde. O preparo foi realizado no Laboratório de Nutrição Experimental da Universidade Federal de Alfenas.

Após o preparo das rações, estas foram acondicionadas hermeticamente em sacos de polietileno, identificados e refrigerados $\left(10-15^{\circ} \mathrm{C}\right)$ até o momento da sua utilização.

Controle de consumo alimentar

Ao longo do experimento os animais receberam aproximadamente $25 \mathrm{~g}$ por dia de dieta, o que é uma quantidade estimada segundo o trabalho de Costa et al. ${ }^{(11)}$ e suficiente para suprir a demanda nutricional dos animais neste período de vida. O consumo alimentar dos quatro grupos experimentais foi contabilizado a partir da pesagem pré-oferta, e no dia seguinte realizouse a pesagem da sobra e do resto. A partir destes valores, determinou-se a estimativa de consumo alimentar. 


\section{Análise da glicemia}

A análise da glicemia dos animais foi realizada no primeiro e no último dia do experimento, utilizando a veia caudal, com fitas de mensuração glicêmica pelo aparelho AccuCheck ${ }^{\circledR}$.

\section{Eutanásia dos animais}

Para proceder à eutanásia, primeiramente os animais foram mantidos em jejum de seis horas. Foram confinados em um dessecador e anestesiados com isoflurano embebido em algodão. Em seguida foram guilhotinados, e posteriormente preparados para a remoção do fígado, ceco e tecido adiposo epididimal com a utilização de materiais cirúrgicos esterilizados.

Análise do peso relativo do ceco e do peso relativo do fígado

Adeterminação do peso relativo do ceco se deu conforme a equação: [peso relativo do $\mathrm{ceco}^{=}$peso do ceco $(\mathrm{g}) \div$ peso corporal final $(\mathrm{g})]$. Para o peso relativo do fígado e quantificação do tecido adiposo epididimal foram adotados o mesmo procedimento. O peso relativo é mais fidedigno quando comparado com o peso absoluto, por levar em consideração o peso corporal. Para a mensuração de todos os pesos foi utilizada uma balança analítica $\left(\right.$ Gehaka $\left.^{\circledR}\right)$.

Análise estatística

As análises estatísticas foram realizadas no software Sigma Stat 4.0 e foram utilizados o teste de normalidade de Shapiro-Wilk, testes descritivos, teste $t$ pareado e ANOVA seguido dos testes de comparação de médias de Tukey. O nível de significância adotado foi de $\mathrm{p}<0,05$.

Aspectos éticos

O presente estudo foi aprovado pela Comissão de Ética para Uso de Animais, da
Universidade Federal de Alfenas/UNIFAL, registro 062/2017. Todos os procedimentos adotados estão conforme o preconizado pelo Colégio Brasileiro de Experimentação Animal.

\section{Resultados}

\section{Composição da farinha e biomassa} de banana verde

Para uma correta averiguação da possibilidade de comparação e o que diferenciaria de maneira geral os ingredientes utilizados, uma vez que estes possuem o mesmo componente biológico ativo (AR), foi realizada a análise centesimal (Tabela 1).

Pode-se observar que as composições centesimais da farinha e da biomassa de banana verde se diferenciam em todos os determinantes analisados, de forma que a farinha de banana verde possui uma maior concentração de todos os componentes da análise de composição centesimal em comparação à biomassa.

Tabela 1 - Análise da composição centesimal da farinha e biomassa de banana verde.

\begin{tabular}{ccc} 
Componente & $\begin{array}{c}\text { Farinha de } \\
\text { banana verde }\end{array}$ & $\begin{array}{c}\text { Biomassa de } \\
\text { banana verde }\end{array}$ \\
\cline { 2 - 3 } & $\%$ & $\%$ \\
\hline Umidade & 7,95 & 78,4 \\
Cinzas & 7,08 & 2,91 \\
Carboidratos & 61,94 & 12,54 \\
Lipídeos & 2,69 & 0,79 \\
Proteína & 4,09 & 1,13 \\
Fibras & 16,25 & 4,23 \\
\hline
\end{tabular}

Fonte: Dados da Pesquisa - Centro de Raízes e Amidos Tropicais - CERAT 2017. 


\section{Teor de amido resistente}

Foi realizada a investigação do teor de amido resistente das amostras de biomassa e farinha de banana verde, uma vez que se torna um dado essencial para a elaboração das dietas utilizadas posteriormente de forma padronizada e com o correto percentual de amido resistente (Tabela 2).

Tabela 2 - Teor de amido resistente das amostras de farinha e biomassa de banana verde.

\begin{tabular}{ccc}
\hline Análise & $\begin{array}{c}\text { Amostra de farinha } \\
\text { de banana verde }\end{array}$ & $\begin{array}{c}\text { Amostra de biomassa } \\
\text { de banana verde }\end{array}$ \\
\hline Amido resistente $(\%)$ & 35,03 & 9,82 \\
\hline
\end{tabular}

Fonte: Dados da Pesquisa - Centro de Raízes e Amidos Tropicais - CERAT 2017.

As diferentes dietas oferecidas (Cafeteria, de cada um dos prebióticos (farinha e biomassa $\mathrm{CAF}+\mathrm{F}$ e $\mathrm{CAF}+\mathrm{B}$ ) aos animais, foram de banana verde) para cada um dos grupos elaboradas baseadas nas recomendações para estudados, respeitando seus respectivos cálculos elaboração de dieta de cafeteria ${ }^{(10)}$ e em seguida de concentrações. A composição da dieta dos cinco adicionados $8 \%$ de amido resistente provenientes grupos experimentais está apresentada na Tabela 3 .

Tabela 3 - Composição $(\mathrm{g} / 100 \mathrm{~g})$ das dietas dos grupos experimentais.

\begin{tabular}{lccc}
\hline Grupos experimentais & CAF & CAF + FV & CAF + BV \\
\hline Biscoito doce recheado & 14,30 & 14,30 & - \\
Batata palha & 14,30 & 14,30 & - \\
Chocolate ao leite & 14,30 & 14,30 & - \\
Bacon & 14,30 & 14,30 & - \\
Patê de sardinha & 28,60 & 19,96 & - \\
Dieta comercial & 14,30 & - & 81,47 \\
Biomassa BV & - & - & - \\
Farinha BV & - & 22,84 & \\
\hline
\end{tabular}

Fonte: Autores

CAF: Grupo controle com dieta de cafeteria; CAF + FV: Grupo de dieta de cafeteria suplementada com farinha de banana verde ( $8 \%$ de amido resistente); CAF + BV: Grupo dieta de cafeteria suplementada com biomassa de banana verde ( $8 \%$ de amido resistente). BV: Banana verde.

\section{Glicemia}

Conforme observado (Tabela 4), houve alteração significativa com menor valor de glicemia após intervenção com a dieta de cafeteria adicionada da farinha de banana verde. No grupo cafeteria houve aumento numérico, e nos demais grupos diminuição numérica - significativa no grupo $\mathrm{CAF}+\mathrm{F}$. 
Tabela 4 - Perfil glicêmico no período basal e pós-intervenção.

\begin{tabular}{cccc}
\hline Grupos experimentais & Período basal & Período pós-intervenção & $\boldsymbol{P}$ \\
\hline $\mathrm{C}$ & $112,33 \pm 8,50^{\mathrm{a}}$ & $101,00 \pm 7,72^{\mathrm{a}}$ & 0,055 \\
$\mathrm{CAF}$ & $107,17 \pm 12,00^{\mathrm{a}}$ & $112,50 \pm 8,48^{\mathrm{a}}$ & 0,375 \\
$\mathrm{CAF}+\mathrm{F}$ & $171,33 \pm 10,91^{\mathrm{a}}$ & $110,00 \pm 7,21^{\mathrm{b}}$ & $<0,001$ \\
$\mathrm{CAF}+\mathrm{B}$ & $113,00 \pm 92,25^{\text {a }}$ & $111,00 \pm 102,75^{\mathrm{a}}$ & 1,000 \\
\hline
\end{tabular}

Fonte: Autores

C: Grupo controle dieta comercial Nuvilab ${ }^{\circledR}$; CAF: Grupo controle com dieta de cafeteria; CAF + F: Grupo de dieta de cafeteria suplementada com farinha de banana verde ( $8 \%$ de amido resistente); CAF + B: Grupo dieta de cafeteria suplementada com biomassa de banana verde ( $8 \%$ de amido resistente).

Médias \pm desvio padrão seguidos de letras diferentes na mesma linha diferem entre si pelo teste $t$ pareado.

De acordo com os dados apresentados (Tabela 5), o consumo alimentar diário diferiu significativamente entre os grupos $\mathrm{C}$ e $\mathrm{CAF}$; $\mathrm{CAF}$ e $\mathrm{CAF}+\mathrm{F}$ e $\mathrm{CAF}+\mathrm{B}$. Nota-se que o ganho de peso foi maior no grupo que recebeu ração comercial e menor no grupo $\mathrm{CAF}+\mathrm{B}$. O peso relativo do ceco e o peso relativo do fígado não apresentaram diferença estatística, o que pode ser em decorrência do período experimental não ter sido suficiente para induzir algumas alterações metabólicas. O tecido adiposo epididimal foi significativamente maior no grupo cafeteria, conforme esperado, e apresentou menor valor no grupo CAF + B. Na Tabela 5 estão dispostos ainda os dados de GP, PRC, PRF e TAE, que serão melhor explorados na discussão.

Tabela 5 - Médias \pm desvio padrão de CAD, GP, PRC, PRF e TAE.

\begin{tabular}{|c|c|c|c|c|c|}
\hline \multirow{2}{*}{ Variáveis } & \multicolumn{5}{|c|}{ Grupos experimentais } \\
\hline & $\mathbf{C}$ & CAF & $\mathbf{C A F}+\mathbf{F}$ & $\mathbf{C A F}+\mathbf{B}$ & $P$ \\
\hline CAD & $16,42 \pm 1,52^{a}$ & $13,71 \pm 2,38^{b}$ & $17,55 \pm 0,21^{\mathrm{a}, \mathrm{c}}$ & $17,69 \pm 1,67^{\mathrm{a}, \mathrm{c}}$ & $0,001^{* 1}$ \\
\hline GP & $88,67 \pm 39,26^{\mathrm{a}}$ & $74,33 \pm 21,01 \mathrm{a}, \mathrm{b}$ & $63,00 \pm 17,72^{\mathrm{a}, \mathrm{b}}$ & $39,83 \pm 11,19^{b}$ & $0,018^{* 2}$ \\
\hline PRC & $0,0061 \pm 0,0050^{a}$ & $0,0035 \pm 0,0027^{\mathrm{a}}$ & $0,0052 \pm 0,0035^{a}$ & $0,0046 \pm 0,0045^{a}$ & $0,170^{* 3}$ \\
\hline PRF & $0,04 \pm 0,006^{\mathrm{a}}$ & $0,04 \pm 0,006^{\mathrm{a}}$ & $0,04 \pm 0,002^{\mathrm{a}}$ & $0,04 \pm 0,004^{\mathrm{a}}$ & $0,503^{* 4}$ \\
\hline TAE & $1,98 \pm 1,62^{\mathrm{a}, \mathrm{b}}$ & $3,07 \pm 2,55^{\mathrm{a}}$ & $2,87 \pm 2,21^{\mathrm{a}, \mathrm{b}}$ & $2,21 \pm 2,07^{b}$ & $0,033^{* 4}$ \\
\hline
\end{tabular}

Fonte: Autores

C: Grupo controle dieta comercial Nuvilab ${ }^{\circledR}$; CAF: Grupo controle com dieta de cafeteria; CAF + F: Grupo de dieta de cafeteria suplementada com farinha de banana verde ( $8 \%$ de amido resistente); CAF + B: Grupo dieta de cafeteria suplementada com biomassa de banana verde ( $8 \%$ de amido resistente). CAD: Consumo alimentar diário; GP: Ganho de peso; PRC: Peso relativo do ceco; PRF: Peso relativo do fígado; TAE: Tecido adiposo epididimal.

${ }^{*}, 2$ Médias \pm desvio padrão seguidos de letras diferentes na mesma linha diferem entre si pelo teste de Tukey;

${ }^{* 3,4}$ Médias \pm desvio padrão seguidos de letras diferentes na mesma linha diferem entre si pelo teste de ANOVA. 


\section{Discussão}

As diferenças observadas na composição centesimal da farinha e biomassa de banana verde podem ser explicadas pelo processo de fabricação da farinha de banana verde, quando é necessária demanda muito maior de matéria-prima, uma vez que este processo exige a desidratação forçada quase por completo da massa de banana verde; isto causa um grande acúmulo dos diversos componentes existentes no alimento, como já exemplificado por Gonçalves et al. ${ }^{(12)}$

$\mathrm{O}$ teor de amido resistente também foi encontrado substancialmente maior na farinha de banana verde. Este resultado também pode ser explicado pelo processo de fabricação que este ingrediente sofre, seguindo o raciocínio já citado. Isto é, o processo de fabricação é um ponto chave na determinação da composição centesimal e de amido resistente deste prebiótico.

As quantidades de amido resistente presentes nas dietas que o possuem foram limitadas e determinadas a $8 \%$, uma vez que um aumento desta quantidade poderia provocar a desestabilização da microbiota intestinal dos animais, levando à diarreia, possível desidratação e de outros sintomas que levariam ao desconforto, e poderiam causar elevação no estresse do animal. Convém notar também que é necessária uma quantidade muito menor de farinha para se atingir a concentração de amido resistente desejada, uma vez que neste produto os componentes se encontram de forma muito mais concentrada, o que já foi previamente discutido. Devido às diferentes qualidades físicas dos compostos, as dietas apresentaram diferentes texturas e firmeza dos pellets.

Entende-se que o grupo $\mathrm{CAF}+\mathrm{F}$ tenha gerado resultados mais relevantes no controle glicêmico, pois estudos mostram que a fibra da FBV está relacionada com o hormônio GLP-1 (glucagon like peptide), que é considerado um potente agente antidiabetogênico. Isto ocorre, pois o GLP-1 estimula a secreção de insulina e inibe a liberação de glucagon, além de retardar o esvaziamento gástrico. Essas reações acarretam a redução da glicemia, obtendo melhor controle glicêmico. Alguns pesquisadores têm avaliado a relação entre a produção de GLP-1 e consumo alimentar e observase que as fibras solúveis altamente fermentáveis, como a fibra encontrada na FBV, por sua ação prebiótica, produzem grandes quantidades de ácidos graxos de cadeia curta, e estes são potentes estimuladores da secreção de GLP-1.(13)

Verificou-se um consumo diário estatisticamente relevante dos grupos, com o menor consumo pertencente ao grupo CAF seguido do grupo Controle e similar entre as dietas de cafeteria modificadas com amido resistente (CAF $+\mathrm{F}$ e $\mathrm{CAF}+\mathrm{B})$. $\mathrm{O}$ reduzido consumo alimentar do grupo CAF pode ser explicado pela má adesão à dieta, uma vez que esta possuía a consistência mais amolecida dos grupos e se diferencia do grupo C, cuja dieta foi provida para os animais desde o momento de climatização. Por sua vez, os grupos $\mathrm{CAF}+\mathrm{F}$ e $\mathrm{CAF}+\mathrm{B}$ podem ter apresentado os maiores valores de consumo, pois eram os grupos (dentre os de dieta modificada) que possuíam maior dureza e textura parecida com as de ração comercial $\left(\mathrm{Nuvilab}^{\circledR}\right)$. Além disso, nota-se a ação do amido resistente induzindo a liberação de GLP1, e influência sobre o ganho de peso dos animais, o que consequentemente aumentaria o consumo para que houvesse a manutenção do balanço energético.

O ganho de peso (GP) dos animais, assim como o esperado pela proposta, demonstrou-se respectivamente maior nos grupos $\mathrm{C}$ e $\mathrm{CAF}$; no entanto, o consumo do grupo $\mathrm{C}$ superou os valores do grupo CAF, sendo possivelmente explicado pela má adaptação dos animais à dieta administrada, o que engloba os fatores discutidos anteriormente. Entre os grupos de dieta de cafeteria modificada $(\mathrm{CAF}+\mathrm{F}$ e CAF $+\mathrm{B})$ notou-se significante redução do ganho de peso dos animais quando comparados aos grupos $\mathrm{C}$ e $\mathrm{CAF}$, o que sugere influência benéfica do amido resistente presente nestas dietas e a influência destes no metabolismo dos animais, como discutido no tópico Glicemia. Entre estes grupos $(\mathrm{CAF}+\mathrm{F}$ e $\mathrm{CAF}+\mathrm{B})$, o suplementado 
com biomassa foi o que apresentou o menor ganho de peso, o que pode ter sido influenciado negativamente pela quantidade de biomassa de banana verde, veiculada pela dieta.

$\mathrm{O}$ peso relativo do ceco (PRC) demonstrou significância estatística $(\mathrm{P}=0,018)$. Foram observados os maiores valores no grupo $\mathrm{C}$ e $\mathrm{CAF}$, indo contra o resultado esperado pelo estudo, uma vez que a maior presença de prebióticos supostamente causaria aumento na fermentação dos alimentos ingeridos e deveria levar ao aumento do ceco dos animais. Isto pode ter sido determinado pelo curto prazo de experimentação e administração das dietas, impedindo com que houvesse a observação do aumento da fermentação pela microbiota intestinal.

Já o peso relativo do fígado (PRF), por sua vez, não apresentou diferença significativa em nenhum dos grupos, sendo descartada a possibilidade de que com este tempo de administração das dietas haveria maior produção e retenção de tecido adiposo nas células hepáticas.

No entanto, quando avaliado o peso do tecido adiposo epididimal (TAE), foi possível observar uma grande diferença entre os grupos. Assim como esperado, o grupo $\mathrm{C}$ apresentou o menor valor, uma vez que sua dieta era a de menor carga lipídica e não possuía os mesmos componentes da dieta ocidentalizada que continham nos grupos cafeteria $(\mathrm{CAF}, \mathrm{CAF}+\mathrm{F}$ e CAF $+\mathrm{B})$. Também se nota que a dieta de cafeteria administrada exclusivamente aos animais foi a de maior influência no ganho de peso de tecido adiposo epididimal, demonstrando o maior valor entre os grupos. Isto é explicado pelas características da dieta ocidentalizada (representada como grupo CAF), com maior concentração de lipídeos e carboidratos simples. No entanto, no grupo de dieta de cafeteria associada ao amido resistente da farinha de banana verde $(\mathrm{CAF}+\mathrm{F})$ foi possível observar uma redução do TAE em relação ao grupo $\mathrm{CAF}$, podendo ser explicado pelos efeitos benéficos da associação do amido resistente à dieta $\mathrm{CAF}$.

Porém, estes efeitos benéficos contra a dieta CAF tornam-se mais acentuados na dieta associada à biomassa de banana verde (CAF + B), como observado (Tabela 5), mesmo quando seu consumo é semelhante, como previamente discutido, isto pode ser explicado por uma aparente melhor influência do amido resistente proveniente da biomassa do que da farinha de banana verde no ganho de peso e de gordura corporal dos animais, demonstrado pelo TAE.

No presente estudo pode-se notar que o consumo de dietas ocidentalizadas, com altos teores de calorias, desequilíbrio de carboidratos e lipídeos, pode acarretar em malefícios à saúde. A formulação da dieta de cafeteria simula uma dieta desequilibrada, pobre em micronutrientes e compostos bioativos. Entende-se que se houver consumo alimentar com composição similar à dieta de cafeteria corriqueiramente, pode ocorrer aumento exacerbado do tecido adiposo visceral, danos cardiometabólicos e quadros de hiperglicemia constante, e a longo prazo prevê-se o desenvolvimento de doenças crônicas não degenerativas.

\section{Conclusão}

Foi possível observar que as matrizes alimentares que contém amido resistente auxiliam no controle do ganho de peso corporal, prevenindo o acúmulo de tecido adiposo, assim como intervêm no manejo dos níveis glicêmicos. Além destas funções, confirmou-se também que, para que os prebióticos aumentem a massa do ceco após a fermentação e geração de ácidos graxos de cadeia curta demanda-se mais tempo de contato com os compostos biologicamente ativos do que o proposto na metodologia.

Em suma, destaca-se que a implementação dos produtos da banana verde na alimentação pode ser de grande valia, considerando os efeitos positivos de seus componentes bioativos, ação prebiótica e a relação custo benefício desta matriz alimentar. A produção de mais estudos científicos faz-se pertinente para confirmar as demais hipóteses que surgem com o tema. 


\section{Referências}

1 Marques ACRM, Gabbiatti GC, Gravena AAF, Amaral, V. Influência das dietas hipercalóricas sobre os parâmetros de obesidade, dislipidemia e hiperglicemia em ratos. Rev Saúde e Pesq. 2015;8(1):55-62.

2 Oliveira JEP, Vencio S, organizadores. Diretrizes da Sociedade Brasileira de Diabetes: 2014-2015 [Internet]. São Paulo: AC Farmacêutica; 2015. [citado 2016 Mar 24]. Disponível em: https://www.diabetes.org.br/ publico/images/2015/area-restrita/diretrizessbd-2015.pdf

3 Mendes FCV, Moreira BMT, Marsiglio GN, Carabelli B, Munhoz AC, Barella LF, et al. Dieta de cafeteria remodela a estrutura da aorta de ratos obesos. SaBios [Internet]. 2013 [citado 2017 Dez 3]; 8(1). Disponível em: http:// revista.grupointegrado.br/revista/index.php/ sabios2/article/view/1158/486

4 Yamaguchi N, Mahbub MH, Takahashi H, Hase R, Ishimaru Y, Sunagawa H. et al. Plasma free amino acid profiles evaluate risk of metabolic syndrome, diabetes, dyslipidemia, and hypertension in a large Asian population. Environ. Health Prev. Med. 2017; 22:35.

5 Xavier HT, Izar MC, Faria JR Neto, Assad MH, Rocha VZ, Sposito AC. et al. V Diretriz Brasileira de Dislipidemias e Prevenção da Aterosclerose. Arq. Bras. Cardiol. [Internet]. 2013 Oct [citado 2017 Dez 3]; 101(4 Supl 1):1-20. Disponível em: http://www.scielo. br/scielo.php?script $=$ sci_arttext\&pid $=$ S0066$782 X 2013004100001 \& \operatorname{lng}=$ en

6 Paula HAA, Abranches MV, Ferreira CLLF. Yacon (Smallanthus Sonchifolius): A Food with Multiple Functions. Food Science Nutr. 2015;55(1):32-40.

7 Association of Official Analytical Chemists. Official methods of analysis. Virginia: AOAC; 2005.

8 Goni I, Garcia-Diz L, Manãs E, Saura-Calixto F. Analysis of resistant starch: a method for foods and food products. Food Chem. 1996; 56(4):445-9.
9 Weaver CM, Martin BR, Story JA, Hutchinson I, Sanders L. Novel fibers increase bone calcium content and strength beyond efficiency of large intestine fermentation. Jagr Food Chem, 2010;58:8952-7.

10 Campión J, Martínez JA. Ketoconazole, an antifungal agent, protects against adiposity induced by a cafeteria diet. Horm Metab Res. 2004;36(7):485-91.

11 Costa NMB, Peluzio MCG, Martino HSD, Henriques GS. Nutrição experimental: teoria e prática. Rio de Janeiro: Rubio; 2014.

12 Gonçalves JQ, Silva MAP, Plácido MC, Silva RM, Moura LC, Souza DG. Secagem da casca e polpa de banana verde (Musa acuminata): propriedades físicas e funcionais da farinha. Global Science Technology. 2016;9(3):62-72.

13 Waszak MN, Ferreira CC. Efeito hipoglicemiante das farinhas de banana verde e de maracujá no controle da glicemia em diabéticos. Cad UniFOA. 2011;6(1).
Recebido em: 27 nov. 2018

Aceito em: 13 abr. 2020 
